Received: 12 April 2018

Accepted: 25 June 2018

Published online: 07 August 2018

ENTIFIC REP

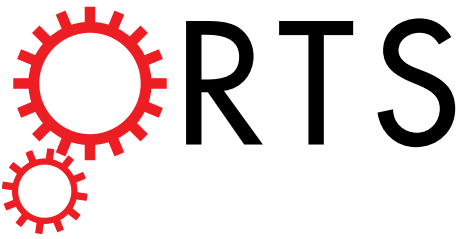

OPEN

\title{
The role of lipids in the inception, maintenance and complications of dengue virus infection
}

\author{
Carlos Fernando Odir Rodrigues Melo $\mathbb{1}^{1}{ }^{1}$, Jeany Delafiori ${ }^{1}$, Mohamad Ziad Dabaja ${ }^{1}$, \\ Diogo Noin de Oliveira ${ }^{1}$, Tatiane Melina Guerreiro ${ }^{1}$, Tatiana Elias Colombo ${ }^{2}$, \\ Maurício Lacerda Nogueira $\mathbb{D}^{2}$, Jose Luiz Proenca-Modena ${ }^{3}$ \& Rodrigo Ramos Catharino ${ }^{1}$
}

Dengue fever is a viral condition that has become a recurrent issue for public health in tropical countries, common endemic areas. Although viral structure and composition have been widely studied, the infection phenotype in terms of small molecules remains poorly established. This contribution providing a comprehensive overview of the metabolic implications of the virus-host interaction using a lipidomicbased approach through direct-infusion high-resolution mass spectrometry. Our results provide further evidence that lipids are part of both the immune response upon Dengue virus infection and viral infection maintenance mechanism in the organism. Furthermore, the species described herein provide evidence that such lipids may be part of the mechanism that leads to blood-related complications such as hemorrhagic fever, the severe form of the disease.

Dengue virus (DENV) is an arbovirus transmitted by mosquitoes of the genus Aedes, such as Aedes aegypti and Aedes albopictus. DENV is associated with outbursts of febrile diseases in the tropics since the 80 's $^{1}$. The large number of DENV-infected patients every year, estimated by the World Health Organization in 390 million, makes DENV the most hazardous arbovirus in the world.

DENV is a series of enveloped viruses belonging to the family Flaviviridae, genus Flavivirus, which are classified in four closely related and antigenically distinct serotypes (DENV-1, DENV-2, DENV-3 and DENV-4). Similary to other flavivirus, the DENV genome consist of a single-stranded positive sense RNA (ssRNA) of almost $11 \mathrm{~kb}$, which encodes a polyprotein that is cleaved into three structural proteins (the capsid (C), the pre-membrane (prM) and the envelope (E) and seven nonstructural proteins, named NS1, NS2A, NS2B, NS3, NS4A, NS4B, and NS5 ${ }^{2}$.

Although the spectrum of clinical outcomes of patients' responses to DENV varies from a subclinical infection to death, the majority of symptomatic patients develop an acute, self-limiting febrile manifestation. Lasting approximately 4-7 days, it is characterized by fever, chills, retro-orbital headache, myalgia, malaise, leukopenia, thrombocytopenia (sometimes severe) and elevated levels of hepatic transaminases ${ }^{3}$. In contrast, a small percentage of infected patients, usually children or adults during a second infection with a different DENV serotype, may develop severe dengue, characterized by spontaneous bleeding, plasma leakage, shock, and organ failure ${ }^{3}$.

The available knowledge indicates that the outcome of DENV infection depends on several factors produced during the beginning of the viral infection such as viral load, presence of non-neutralizing antibodies, immune cells recruitment and production of immune mediators ${ }^{2}$. These factors determine whether the environment is favorable or unfavorable for disease progression by either controlling the viral infection or impairing inflammatory reaction, associated with vascular permeability. Nevertheless, the lack of reliable immunological and other metabolic markers for either protective or pathological responses still an important gap that hinders the development of new diagnostic or prognostic tests or vaccine candidates ${ }^{4,5}$.

Within this context ${ }^{4,5}$, this work aimed to verify the changes in serum lipidome of patients infected with DENV-4, since lipids have been shown to be of great importance in the viral infection process ${ }^{6-8}$. Although the lipid profile of patients infected with DENV has already been established in other studies, most of them performed using liquid chromatography coupled to mass spectrometry (LC-MS) techniques ${ }^{9-11}$, whereas the present

${ }^{1}$ INNOVARE Biomarkers Laboratory, School of Pharmaceutical Sciences, University of Campinas, Campinas, Brazil. ${ }^{2}$ School of Medicine from São José do Rio Preto (FAMERP), São José do Rio Preto, Brazil. ${ }^{3}$ Laboratory of Study of Emerging Viruses (LEVE), Department of Genetic, Evolution and Bioagents, Institute of Biology, University of Campinas, Campinas, Brazil. Correspondence and requests for materials should be addressed to R.R.C. (email: rrc@g.unicamp.br) 


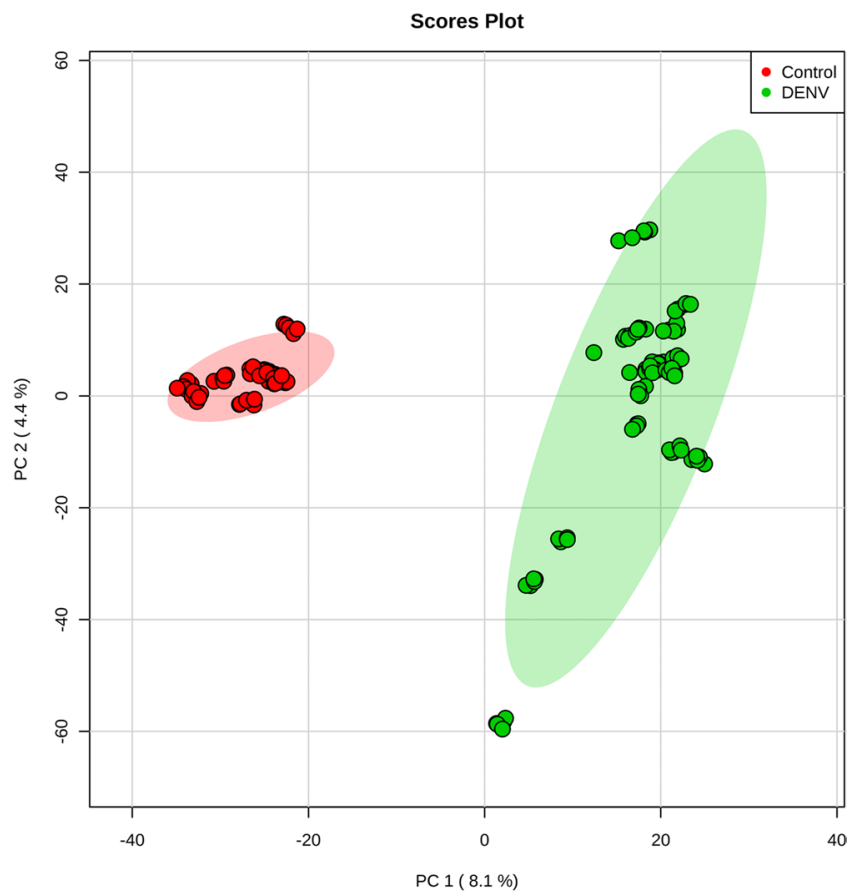

Figure 1. Scores plot between the first two principal components (PCs) selected from the Principal Component Analysis.

study used no chromatographic approach. We intended to analyze samples with the least possible preparation and manipulation, attempting to minimize as much as possible changes in the biological matrix used. Additionally, direct infusion high-resolution mass spectrometry allows us to analyze a wide range of lipids, a characteristic that is impaired when using LC-MS, since the column separates lipids by their respective physicochemical characteristics such as polarity, isomerism and others ${ }^{12,13}$.

\section{Metabolomic approach of serum of patients infected with DENV-4}

PCA clearly shows the separation between Control and DENV-infected patients, as shown in Fig. 1. This multivariate data analysis method was chosen because it is an unsupervised approach, capable of reducing the number of variables (reduction of dimensions) in the original dataset (raw data) based on the similar features between the samples, helping to find the most representative variables (features) responsible for each of the two clusters formed $^{14}$, according to Fig. 1. This enabled the election of a feature set that is characteristic for each analyzed group, namely DENV-infected patients and healthy individuals. The bidimensional score plot in Fig. 1 is derived from the analysis performed with the data collected by mass spectrometry in the positive mode; from this clustering, we selected and characterized the features (potential metabolomic markers) that presented the greatest relevance in discriminating both groups. To illustrate the characteristic markers chosen by PCA, a heatmap of all features selected by this model was built using Pearson's distance measurement and Ward's clustering algorithm (Fig. 2).The Fig. 2 clearly illustrates the differences in the pool of metabolites between both analyzed groups. Following structure elucidations by mass accuracy and MS/MS reactions ${ }^{15}$ (Supplementary Information 1), three precursors of Platelet Activation Factor (PAF) $[\mathrm{m} / z$ 768.5917, $m / z 770.6043$ and $\mathrm{m} / z$ 792.5917], three Phosphatidylcholine derivatives (PC) $[\mathrm{m} / z 762.6022, \mathrm{~m} / z 838.6336$ and $\mathrm{m} / z 796.6231]$ and four triglycerides $[\mathrm{m} / z$ $743.6169, \mathrm{~m} / z$ 769.6327, $\mathrm{m} / z$ 795.6412 and $\mathrm{m} / z$ 859.7765] were identified as characteristic for the DENV group; a list of characterized molecules is available in Table 1 . Moreover, by elucidating relevant species such as the above mentioned, this work has confirmed that it is possible to identify in vivo which are the lipids associated with the phenotype of viral infection process by directly infusing the serum of infected patients in an HRMS instrument, regardless of any previous chromatographic approach.

\section{Compounds involved in the pathophysiological mechanism of DENV-4 infection}

Our findings corroborate previous reports by other groups, in studies that identified the production of a series of polypeptides that act as inflammatory mediators during the immune response of the host ${ }^{3}$, participating in the autophagy process by signaling between virus and host cell ${ }^{2}$ and in their replication process ${ }^{16}$. These studies have also reported the synthesis of lipid mediators during viral infection, which are related to the signaling, control, and maintenance of both the immune response and DENV pathogenesis ${ }^{6,17-19}$.

\section{Phosphatidylcholines (PC) and Triglycerides (TG)}

The importance of lipid changes during DENV cell infection is evident when the virus assumes control of the cellular metabolism by controlling and regulating autophagy mechanisms to meet the needs of the viral replication ${ }^{20}$. 


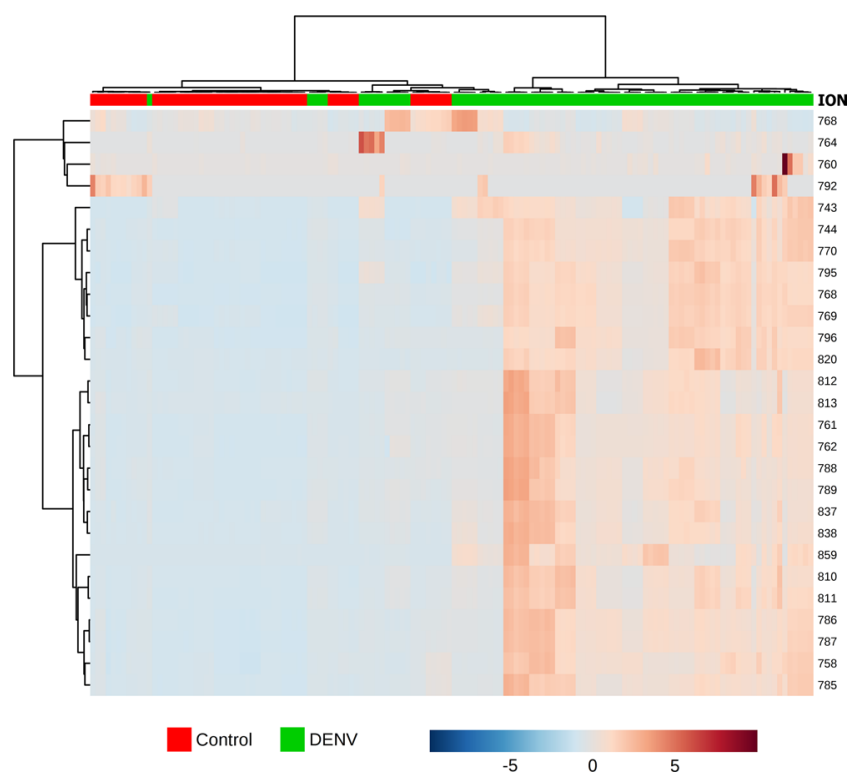

Figure 2. Clustering result for the 27 top features selected by Principal Component Analysis shown as a heatmap (distance measured by Pearson's distance measurement and Ward's clustering algorithm). The colorcoded thermometer (bottom) indicates the relative presence of metabolites among the groups.

\begin{tabular}{|c|c|c|c|c|c|c|c|}
\hline Exact mass & Theoretical Mass & Error $(\mathrm{ppm})^{\mathrm{a}}$ & MS/MS fragmentation & Adducts & Platform $^{\mathrm{b}}$ & Molecule & $\log 2(\mathrm{FC})^{\mathrm{c}}$ \\
\hline \multirow{2}{*}{743.6169} & \multirow{2}{*}{743.6184} & \multirow{2}{*}{2.0172} & \multirow{2}{*}{$684,619,555,487$} & \multirow{2}{*}[\mathrm{M}+\mathrm{H}]{+} & MID 98508 & \multirow{2}{*}{ TG(44:4) } & \multirow{2}{*}{4.245} \\
\hline & & & & & MID 99076 & & \\
\hline \multirow{2}{*}{769.6327} & \multirow{2}{*}{769.6341} & \multirow{2}{*}{1.8190} & \multirow{2}{*}{$709,645,581,587$} & \multirow{2}{*}[\mathrm{M}+\mathrm{H}]{+} & MID 99084 & \multirow{2}{*}{ TG(46:5) } & \multirow{2}{*}{2.9707} \\
\hline & & & & & MID 98516 & & \\
\hline \multirow{2}{*}{795.6412} & \multirow{2}{*}{795.6424} & \multirow{2}{*}{1.5082} & \multirow{2}{*}{$736,612,607,590$} & \multirow{2}{*}[\mathrm{M}+\mathrm{H}]{+} & MID 99092 & \multirow{2}{*}{ TG(48:6) } & \multirow{2}{*}{3.7961} \\
\hline & & & & & MID 98531 & & \\
\hline 859.7765 & 859.7749 & 1.8610 & $799,676,842,671$ & {$[\mathrm{M}+\mathrm{H}]+$} & MID 4798 & TG(52:2) & 7.4812 \\
\hline \multirow{5}{*}{762.6022} & \multirow{5}{*}{762.6007} & \multirow{5}{*}{1.9670} & \multirow{5}{*}{$575,704,621,719$} & \multirow{5}{*}[\mathrm{M}+\mathrm{H}]{+} & MID 59328 & \multirow{5}{*}{$\mathrm{PC}(34: 0)$} & \multirow{5}{*}{2.6625} \\
\hline & & & & & MID 39142 & & \\
\hline & & & & & MID 59482 & & \\
\hline & & & & & MID 59708 & & \\
\hline & & & & & MID 39823 & & \\
\hline \multirow{2}{*}{784.5836} & \multirow{2}{*}{784.5851} & \multirow{2}{*}{1.9118} & & & MID 59843 & & \\
\hline & & & $579,595,725,601$ & {$[\mathrm{M}+\mathrm{H}]+$} & MID 59614 & PC(36:0) & 8.9199 \\
\hline & & & & & MID 59917 & & \\
\hline 838.6336 & 838.6320 & 1.9079 & $779,649,655,721$ & {$[\mathrm{M}+\mathrm{H}]+$} & MID 59982 & $\operatorname{PC}(40: 0)$ & 4.8301 \\
\hline & & & & & MID 39855 & & \\
\hline & & & & & MID 43414 & & \\
\hline 768.5917 & 768.5902 & 1.9516 & $709,581,585,563$ & {$[\mathrm{M}+\mathrm{H}]+$} & MID 40083 & $\mathrm{PC}(\mathrm{O}-36: 4)$ & 3.2534 \\
\hline & & & & & MID 76437 & & \\
\hline & & & & & MID 76435 & & \\
\hline 770.6043 & 770.6058 & 1.9465 & $711,583,726,567$ & {$[\mathrm{M}+\mathrm{H}]+$} & MID 40080 & $\mathrm{PC}(\mathrm{O}-36: 3)$ & 3.6014 \\
\hline & & & & & MID 43415 & & \\
\hline 7925917 & 7925902 & 18925 & 733.416609605 & {$[\mathrm{M}+\mathrm{Hl}+$} & MID 62936 & $P C(O-38.6)$ & 27396 \\
\hline 192.591/ & 192.5902 & 1.8925 & $733,416,609,605$ & {$[\mathrm{M}+\mathrm{H}]+$} & MID 40092 & $P C(0-38: 6)$ & 2.7396 \\
\hline & & & & & MID 76462 & & \\
\hline 796.6231 & 796.6215 & 2.0085 & $737,613,778,752$ & {$[\mathrm{M}+\mathrm{H}]+$} & MID 76423 & $\mathrm{PC}(\mathrm{O}-38: 4)$ & 2.9874 \\
\hline & & & & & MID 40129 & & \\
\hline
\end{tabular}

Table 1. Lipid markers elected by Principal Component Analysis from the serum of patients infected with DENV (DENV group). ${ }^{a}$ Error $=(($ Exact Mass-Theoretical Mass $) /$ Exact mass $) * 10^{6} .{ }^{b}$ METLIN ID ${ }^{15} .{ }^{c} \log _{2}($ FC): Log2 transformed fold change where $\mathrm{FC}=$ Fold Change (DENV/Control) prior normalization. 
Autophagy is a general term used to refer to pathways by which cytoplasmic material (soluble macromolecules and organelles) are delivered to the lysosomes for degradation ${ }^{21}$. There are three different processes characterized by autophagy: macroautophagy, autophagy mediated by chaperones, and microautophagy ${ }^{21}$. By these processes, an eukaryotic cell is able to promote essential lysosomal degradation for survival, differentiation, development and homeostasis, presenting an important adaptive role in the protection of organisms against several pathologies $^{22}$. Eliminating defective proteins and organelles with the potential to trigger pathogenic processes prevents the abnormal accumulation of protein aggregates and the removal of obligate intracellular parasites (OIPs); additionally, the autophagic process also plays an important role in the innate and adaptive immunity: it is responsible for the formation of epitopes presented by MHC complexes ${ }^{23}$. Autophagy is rapidly and positively regulated by cells that need to obtain intracellular nutrients, either during a period of nutrient deprivation or absence of growth factors, as well when there is high energy demand ${ }^{22}$. In this way, DENV, like any other virus, controls the cellular mechanisms in its favor ${ }^{20}$. By taking control of the autophagy processes, the virus is able to control cellular lipid metabolism ${ }^{24}$, providing the demands required in the viral infection process ${ }^{20}$.

Viral growth occurs through the formation of viral replication complexes (VRCs) ${ }^{25}$, consisting of lipid vesicles constructed by all positive-strand RNA viruses from the reorganization of the host intracellular membranes; within this vesicle, viral assembly occurs ${ }^{26}$.

Zhang, Jiantao, et al. (2016) demonstrated that a significant increase in PC is associated with viral replication, and occurs mainly in the perinuclear membrane of the endoplasmic reticulum (ER), where viral replication occurs; additionally, their data showed that PC accumulation is due to the formation of this lipid class at the region where the VRC will remain, and not due to the transport of preexisting PC in the cellular interior ${ }^{25}$. Thus, the 3 PCs identified herein as characteristic molecules for the group of infected patients (Table 1) are putatively related to the pool of PCs synthesized by the infected cells for viral replication. These data are not only in agreement with the role of PCs during viral infection by positive-chain RNA viruses ${ }^{6,11,25}$, but also demonstrate that the results obtained by direct in vitro analysis are corroborated in vivo.

Since DENV controls the lipid metabolism of the host cell ${ }^{20,24}$, and there is a urge for an additional bioenergetic demand in the viral replication process, the virus promotes the mobilization and recruitment of lipid droplets responsible for the cellular stock of TGs and cholesteryl esters ${ }^{27}$. Recruited TGs undergo the action of lipases in order to provide the necessary fatty acids (FA) for the additional energy supply, since these TGs are used for the production of ATP through the $\beta$-oxidation pathway ${ }^{28}$. Cholesterol, also released during this process, will be used to form VRC for viral replication, as well as $\mathrm{PCs}^{29-31}$. Thus, TGs identified in this study (Table 1) were indicated as markers present in serum of patients infected with DENV, since the increased bioenergetic demand for viral replication leads to a higher recruitment of TGs as an energy source ${ }^{24}$.

\section{The role of platelet activation factor in DENV infection}

Platelet activation factor (PAF) is the trivial name of a phospholipid that has the chemical structure of 1-O-alkyl2-acetyl-sn-glycero-3-phosphocholine, characterized by an alkyl ether bond at the sn-1 position in the glycerol chain (Fig. 3) ${ }^{32}$. The hexadecyl (16:0) moiety as the linker at the sn-1 position provides greater biological activity to the PAF; however, chain length specificity is low, and this leads to the natural formation of a significant amount of 1-O-octadecyl species, i.e. with an octadecyl (18:0) moiety as the linker at the sn-1 position ${ }^{33}$. Given the variation in the length of the side chain of $\mathrm{O}$-alkyl bound at the sn-1 position, as well as the variation of the 2 -acetyl chain attached at the sn-2 position, a series of different PAFs may be formed at the same time ${ }^{34,35}$. The formation of PAFs occurs either by de novo synthesis, or by the lipid remodeling pathway ${ }^{36}$. The formation of PAFs by de novo synthesis is related to the maintenance of the physiological concentrations of this mediator when the organism is in homeostasis, i.e. constitutive PAF concentrations ${ }^{37}$. The synthesis of PAFs from the remodeling pathway, on the other hand, is regulated by extracellular stimuli, i.e. under inflammation or infection, and is responsible for the PAF pool that occurs under these conditions ${ }^{38,39}$. The synthesis of a pool of PAFs occurring under infection/inflammation is mediated by the activation of cytosolic PLA2 (cPLA2), which recruits

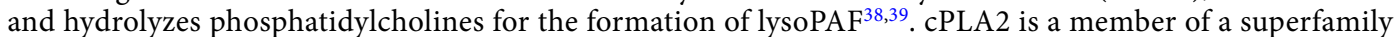
of phospholipases responsible for the recruitment of a series of lipids involved in inflammatory and immune response processes ${ }^{10}$. Therefore, with the activation of cPLA2 due to extracellular stimuli (phosphorylation and $\mathrm{Ca}^{2+}$ ), PAFs synthesis is initiated by the remodeling pathway, with the formation of 1-O-alkyl-sn-glycer3-phosphocholine (lyso-PAF) from the hydrolysis of 2-Acyl-1-alkyl-sn-glycero-3-phosphocholine by cPLA2, which has the characteristic of hydrolyzing fatty acids linked to the sn- 2 position of the glycerol chain ${ }^{37}$. Thus, the action of the platelet-activating factor acetylhydrolase, activated by $\mathrm{Ca}^{2+}$, and phosphorylation of lyso- $\mathrm{PAF}^{40}$, leads to PAF formation (Fig. 3).

PAFs exhibit biological activity in various cells and tissues ${ }^{41}$ and the interaction of PAF agonists occur through the PAF receptor (PAFR). PAFR is comprised of seven transmembrane helixes coupled to the G protein, and is present in both plasma membrane and nuclear membrane. Activation of this receptor leads to the influx of $\mathrm{Ca}^{2+}$ into the cell, promoting a series of simultaneous signaling of kinases and phospholipases, such as MAPK, PKC, phosphatidylinositol-3-kinase (PI3K), protein tyrosine kinase (PTK), phospholipase C $\beta$ (PLC $\beta$ ), and PLA ${ }^{42}$. Thus, cPLA2 itself is activated by increasing $\mathrm{Ca}^{2+}$ concentrations and phosphorylations promoted by the interaction of PAF with its receptor, generating a positive feedback for the production of a PAF pool ${ }^{40,43}$.

The simultaneous activation cascade resulting from PAF promotes a multitude of effects involved in the immune and inflammatory responses, such as the production of superoxide, thromboxane B2 and leukotriene $\mathrm{C} 4^{44}$, increased endothelial permeability ${ }^{45}$, increased production of proinflammatory interleukins ${ }^{46}$, eosinophils recruitment ${ }^{47}$, among other effects involved in the immune response against viral infection. PAFR is present in the membranes of various cell types such as eosinophils, leukocytes, macrophages, neutrophils and platelets ${ }^{43}$. The correlation between PAF and platelet activation is important in the viral infection process, especially for DENV; 


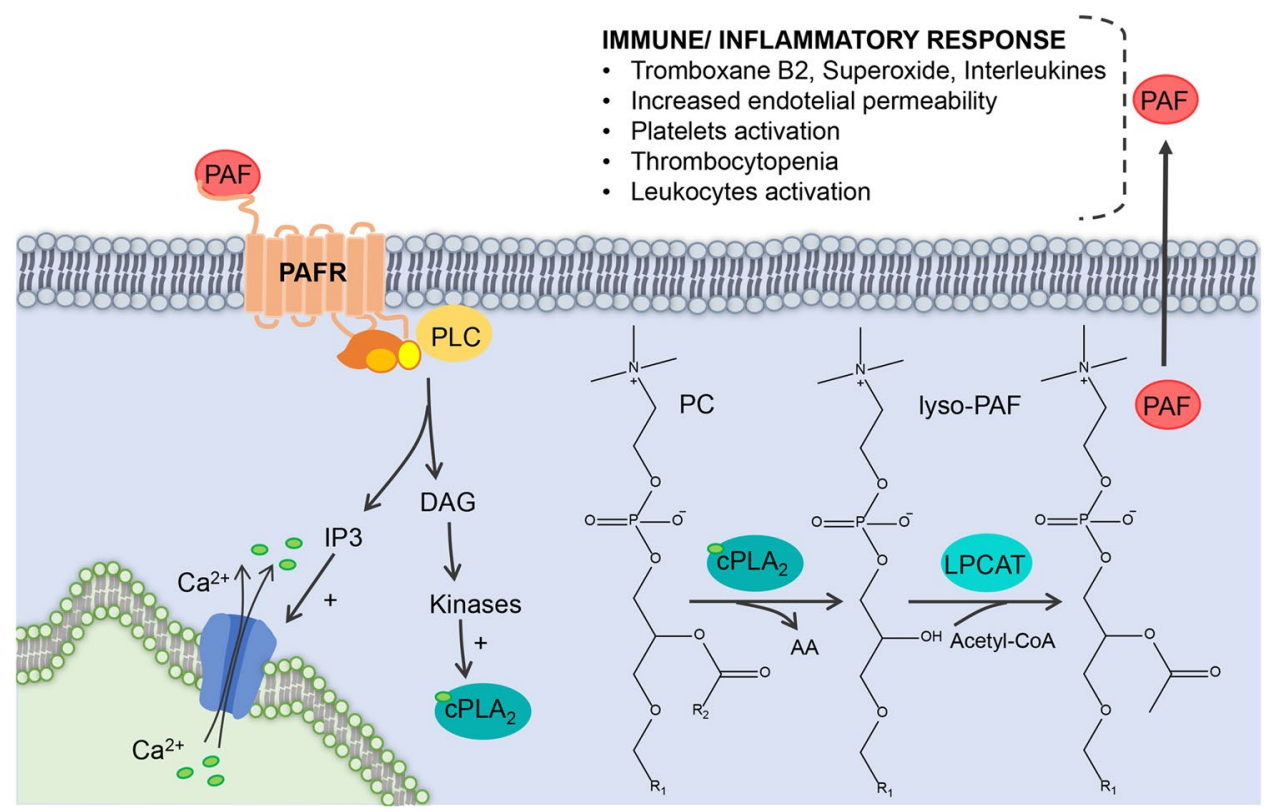

Figure 3. The pool in the synthesis of PAFs that occurs under infection it is mediated by the activation of cPLA2, which recruits and hydrolyzes phosphatidylcholines for the formation of lysoPAF. The activation of cPLA2 due to extracellular stimuli (intracellular phosphorylation and Ca2+ influx) upon PAFs synthesis is initiated by the formation of lyso-PAF. Also, the action of the PAF acetylhydrolase, activated by $\mathrm{Ca} 2+$, and phosphorylation of lyso-PAF leads to PAF formation. PAF, Platelet activation factor; PAFR, Platelet activation factor receptor; PLC, Phospholipase; DAG, Diacylglycerol; IP3, Inositol trisphosphate; Ca2+, Calcium ions; cPLA2, Cytosolic phospholipase A2; PC, Phosphatidylcholine; AA, Arachidonic acid; Lysp-PAF, 1-O-alkyl-snglycer-3-phosphocholine; LPCAT, Lysophosphatidylcholine acyltransferase; Acetyl-CoA, Acetyl coenzyme A.

the endogenous release of PAF is related to a number of acute inflammation effects in DENV infection, such as increased vascular permeability, altered leukocyte numbers, thrombocytopenia, and degrees of bleeding ${ }^{48}$. Thus, the identification of 4 PAF precursors as biomarkers becomes a strong indication for representing this process.

It has recently been shown that platelets are also involved in the detection of pathogen-associated molecular patterns (PAMPs) ${ }^{49}$ by standard-recognition receptors (PRRs) on the surface, such as Toll-like receptors (TLRs), and associated with other members of the interleukin-1 (IL-1)-TLR superfamily. Moreover, it has been reported that the number of constitutive PRR on the surface of the platelet undergoes specific upregulation when the platelet is active by a given microorganism ${ }^{50}$, which would increase the sensitivity to the immune response to the pathogen, assisting in the fight against infection. This mechanism indicates that platelets are part of the first-line recognition process for both microbial pathogens and immune response. Given the fact that platelets have direct antimicrobial functions mediated by the secretion of antimicrobial molecules, including platelet microbicidal proteins (PMPs) and kinocidins ${ }^{49}$; Tsegaye et al. 2013 demonstrated that the release of CXCL4 by platelets inhibited in vitro HIV-1 infection in T cells. Thus, it is feasible to infer that platelet activation may play an important role during the viral immune response process ${ }^{51}$.

The identification of precursor species involved in the synthesis of PAFs in this study is in line with the findings proposed by Berthet et al. 2012, where peripheral blood platelets exposed to S. minnesota led to increased concentrations of IL-6, IL-8 and TNF $\alpha$, whereas platelets exposed to $E$. coli did not ${ }^{52}$. This behavior evidences that the secretion of platelet cytokines is distinct due to the activation pattern, and suggests a specific response where lipids are responsible for such specificity ${ }^{53,54}$.

In contrast to helping fight viral infections, one of the major complications manifested as a result of the interaction between viral infection and platelets, and which is directly related to the activation of PAF, is thrombocytopenia $^{55,56}$. This characteristic manifestation of DENV infection is even used as one of the criteria for the diagnosis of this infection ${ }^{56}$. Thrombocytopenia has been used as a parameter for the identification of patients with a more severe clinical picture, which also encompass other symptoms such as increased vascular permeability and hematocrit, alterations in the number of leukocytes and hemorrhage (varying degrees) ${ }^{48}$. A study by Yang et al. demonstrated greater release of PAF by macrophages in patients with DENV-1 virus than control subjects $^{57}$. In addition, in studies with mice deficient in PAFR, primary infection by DENV was less severe. This occurs because inhibition of the PAF-PAFR interaction decreases the production of proinflammatory cytokines and TNF- $\alpha$, in addition to decreasing vascular permeability ${ }^{57,58}$. The increase in intracellular $\mathrm{Ca}^{2+}$, which may be promoted by the PAF-PAFR interaction, is a crucial factor in the activation of platelet response, including the translocation of P-selectin to the membrane ${ }^{59}$.

Thrombocytopenia in DENV infection occurs due to the immunological destruction of virus-platelet complexes; viral activation of platelets induces overexpression of P-selectin, functioning as a receptor for macrophages ${ }^{60}$. In cases of recurrent DENV infections, antibodies against the prM structural viral protein facilitate 


\begin{tabular}{|l|l|l|}
\hline \multirow{2}{*}{ Parameters } & Groups \\
\cline { 2 - 3 } & Control & DENV \\
\hline RT-PCR exam & Negative & Positive \\
\hline Symptomatic & No & Yes \\
\hline Demographics & 6 & 8 \\
\hline Male & 4 & 12 \\
\hline Female & $42.76(30)$ & $41.4(42)$ \\
\hline Mean age (median) & \multicolumn{2}{|l|}{} \\
\hline Days between onset of DENV symptoms disease and sample collection \\
\hline Day 0 & NA $^{\mathrm{a}}$ & 6 \\
\hline Day 1 & NA $^{\mathrm{a}}$ & 5 \\
\hline Day 2 & NA $^{\mathrm{a}}$ & 5 \\
\hline Day 3 & NA $^{\mathrm{a}}$ & 4 \\
\hline
\end{tabular}

Table 2. Demographics and clinical conditions of all recruited and included individuals in the study. ${ }^{\mathrm{a} N A}$ : Not applicable.

efficient binding of their immature particles to cells expressing the Fc receptor, such as platelets (FccRIIa), which bind to these anti-prM-DENV complexes susceptible to destruction by the immune system ${ }^{61}$. In addition, the DENV-platelet complex also binds to complement C3 molecules and to platelet-associated IgM or IgG antibodies, resulting in their clearance by immune system cells ${ }^{62}$, which would result in thrombocytopenia. Additionally to the mechanisms of destruction of the platelet-DENV complex mentioned above, anti-DENV antibodies react against platelet glycoproteins mediating their destruction by the complement or monocyte-macrophage system ${ }^{63}$.

Therefore, all PAF precursors identified and elucidated in this study highlight the importance of increasing the synthesis of PAF performed by the remodeling pathway in the inflammatory process, confirming its activation by external factors ${ }^{36}$. In addition, the synthesis of PAF pools represents the extensive activation of PAFR, a factor related to the severity of dengue cases, which leads to increased cytokines, increased vascular permeability and, consequently, severe hemorrhage and shock ${ }^{48}$. Moreover, platelet activation correlates with the initiation and maintenance of the immune response, as platelets participate in the front line detection and initiation of the immune response ${ }^{49}$. Platelets still play an ambiguous role in the literature, in which they collaborate in the fight against the infection in the organism ${ }^{49}$, while at the same time are involved in the degree of severity of the disease, being responsible for provoking hemorrhagic fever due to thrombocytopenia ${ }^{56}$.

A previous contribution by our research group has confirmed that it is possible to carry out a study on viral infection mechanism through the direct analysis of the serum of infected patients ${ }^{7}$, assertively providing the metabolomics profile of the pathophysiology of the viral infection process, without further degrees of sample preparation and (pre-)processing. Remarkably, this is possible thanks to the integration between mass spectrometry and bioinformatics to analyze the large amount of data generated. All biomarkers were chosen and validated by statistical analysis and are in line with previous studies on the changes arising from DENV infection both in vivo and in vitro ${ }^{19,20,25,64}$. Thus, the use of these biomarkers opened the possibility to systemically assess the alterations on the lipid pool due to DENV infection, which occurred through the increase of PC synthesis and the recruitment of TGs to supply the bioenergetic needs due to the infection. This study also clarifies the possible mediators of the most severe form of the disease, the hemorrhagic form, since the direct analysis of serum allowed to identify a series of precursors of PAFs. According to data in the literature, the increase in PAFs is closely related to two of the main characteristics of this infection: hemorrhagic fever ${ }^{16}$ and thrombocytopenia ${ }^{59}$. These symptoms, nonetheless, are not exclusively a result of DENV infection: they occur in other infectious diseases as well ${ }^{55,56,61}$. Hence, future efforts in identifying metabolites directly related to several infection processes, as performed herein, will allow us to verify whether the mechanism involved in these diseases is common or specific for each pathogen. Also, our results may enables and encourages the medical community to screen patients with conditions that have potential for hemorrhagic aggravations with a higher degree of confidence for clinical prognosis.

\section{Methods}

Patients. In this study, serum samples stored in the Research Laboratory of Virology from the Faculty of Medicine of São José do Rio Preto (SJRP), a city located in the northwestern region of São Paulo State, Brazil, were analyzed. All 20 sera samples infected with DENV were obtained from febrile patients serviced in healthcare centers in SJRP during the year of 2014, when Zika virus and Chikungunya virus were not detected in São Paulo State. The control group was composed of 10 healthy adults, i.e. asymptomatic individuals who did not present any signs of infection within 30 days prior to sample collection and presented a negative result in RT-PCR for DENV. All sera were transported in dry ice to the INNOVARE Biomarkers Laboratory in Campinas, SP. This study was conducted according to the Declaration of Helsinki and was approved by the Ethics Committee from the Faculty of Medicine of SJRP (FAMERP), São José do Rio Preto, São Paulo, Brazil (Process Number $n^{\circ}$ 02078812.8.0000.5415). The collected specimens from all participants consisted of blood samples. Table 2 organizes the structure of sample collection and provides a view of the total number of analyzed specimens. A written informed consent was obtained from all patients prior to enrollment. All samples were obtained from the Base Hospital of SJRP. All experiments were performed in accordance with relevant guidelines and regulations regarding samples from human origin. 
DENV detection. All clinical samples used in this study were positive for DENV-NS1 antigen using the NS1 Ag rapid assay kit according to the manufacturer's instructions. In addition, all samples were positive for DENV by a specific RT Multiplex-Nested-PCR performed after RNA extraction from $140 \mathrm{uL}$ of serum with the QIAamp Viral RNA mini kit (QIAGEN), according to the manufacturer's protocol. The Multiplex-Nested-PCR to DENV 1-4 were performed according Colombo and collaborators, $2016^{65}$.

Sample preparation. Serum preparation was performed as described by Melo et al. $2017^{7}$. In summary, $20 \mu \mathrm{L}$ of each biological sample (blood serum) were diluted in $200 \mu \mathrm{L}$ of tetrahydrofuran and homogenized and then the volume was completed to $1 \mathrm{~mL}$ with methanol, with further homogenization. The obtained solution was centrifuged and $20 \mu \mathrm{L}$ of the supernatant was collected and diluted in $980 \mu \mathrm{L}$ of methanol and $0.1 \%$ of formic acid was added to the final solution.

High Resolution Mass Spectrometry Analysis. All samples were directly injected for survey scan analysis in an ESI-LTQ-XL Orbitrap Discovery instrument (Thermo Scientific, San Jose, California) with nominal resolution of 30,000 (FWHM), under the following parameters: flow rate of $10 \mu \mathrm{L} \cdot \mathrm{min}^{-1}$, capillary temperature of $280^{\circ} \mathrm{C}, 5 \mathrm{kV}$ as spray voltage and sheath gas at 10 arbitrary units. HRMS analyses were performed in technical quintuplicates for each sample using the mass range of $500-2000 \mathrm{~m} / \mathrm{z}$ in the positive ion mode. Spectra were analyzed using XCalibur software (v. 2.4, Thermo Scientific, San Jose, CA)

Statistical analysis. Statistical analysis to choose chemical markers for each group was performed using Principal Component Analysis (PCA). PCA is a multivariate model of covariance structure modeling; it is used with the specific purpose of analyzing correlation structures, and it is characterized as a statistical analysis technique for potential biomarkers screening by a given "omic" platform ${ }^{14}$. To perform PCA analyses, raw data were used as a pool of all samples within the same data matrix, i.e. all mass spectrometric data from all replicates of both Control and DENV group were organized in a single database, which was inputted in the online platform environment. Prior to PCA analyses, interquartile range was used as data filtering method, with quantile normalization and auto scaling. A heatmap of the all features selected by PCA analyses was built using the Pearson's distance measurement and Ward's clustering algorithm. Fold Change analysis was performed for all features selected by PCA and elucidated by HRMS and MS/MS analysis. All statistical analyses were performed using the online platform MetaboAnalyst 3.0 $0^{66,67}$.

Identification of markers. METLIN (Scripps Center for Metabolomics, La Jolla, CA) was consulted to elect the most suitable markers based on the exact mass of each species, adopting a maximum error of $2 \mathrm{ppm}$ for mass accuracy from the experimental exact mass obtained in the study and adducts of $\left[\mathrm{M}+\mathrm{H}^{+}\right]$and $\left[\mathrm{M}+\mathrm{Na}^{+}\right]$ available on the platform ${ }^{15}$. The markers selected on METLIN were confirmed across MS/MS data acquired in the same instrument used for the HRMS analyses and with the same setup. MS/MS reactions were carried out using Helium as the collision gas, with energies for collision-induced dissociation (CID) ranging from 16 to 31 (arbitrary units). The fragmentation analysis profile spectra of MS/MS were analyzed using XCalibur software (v. 2.4, Thermo Scientific, San Jose, CA) and structures were confirmed using theoretical calculations modeling for molecular fragmentation using Mass Frontier software (v. 6.0, Thermo Scientific, San Jose, CA) (Table 1 and Supplementary Information 1).

\section{References}

1. Simmons, C. P. et al. Dengue. N. Engl. J. Med. 366, 1423-32 (2012).

2. Diamond, M. S. \& Pierson, T. C. Molecular Insight into Dengue Virus Pathogenesis and Its Implications for Disease Control. Cell 162, 488-492 (2015).

3. Rothman, A. L. Immunity to dengue virus: A tale of original antigenic sin and tropical cytokine storms. Nature Reviews Immunology 11, 532-543 (2011).

4. Guzman, M. G. et al. Dengue: A continuing global threat. Nat. Rev. Microbiol. 8, S7-S16 (2010).

5. Amâncio, F. F. et al. Fatal Outcome Of Infection By Dengue 4 In A Patient With Thrombocytopenic Purpura As A Comorbid Condition In Brazil. Rev. Inst. Med. Trop. Sao Paulo 56, 267-270 (2014).

6. Melo, C. F. O. R. et al. A lipidomics approach in the characterization of zika-infected mosquito cells: Potential targets for breaking the transmission cycle. PLoS One 11 (2016).

7. Melo, C. F. O. R. Serum Metabolic Alterations upon ZikaInfection. Front. Microbiol. 8 (2017).

8. Nolte-'t Hoen, E., Cremer, T., Gallo, R. C. \& Margolis, L. B. Extracellular vesicles and viruses: Are they close relatives? Proc. Natl. Acad. Sci. 113, 9155-9161 (2016).

9. Soto-Acosta, R. et al. The increase in cholesterol levels at early stages after dengue virus infection correlates with an augment in LDL particle uptake and HMG-CoA reductase activity. Virology 442, 132-147 (2013).

10. Chandrasekharan, J. A., Marginean, A. \& Sharma-Walia, N. An insight into the role of arachidonic acid derived lipid mediators in virus associated pathogenesis and malignancies. Prostaglandins and Other Lipid Mediators 126, 46-54 (2016).

11. Perera, R. et al. Dengue virus infection perturbs lipid homeostasis in infected mosquito cells. PLoS Pathog. 8 (2012).

12. Ortmayr, K., Hann, S. \& Koellensperger, G. Complementing reversed-phase selectivity with porous graphitized carbon to increase the metabolome coverage in an on-line two-dimensional LC-MS setup for metabolomics. Analyst 140, 3465-3473 (2015).

13. Matuszewski, B. K., Constanzer, M. L. \& Chavez-Eng, C. M. Strategies for the assessment of matrix effect in quantitative bioanalytical methods based on HPLC-MS/MS. Anal. Chem. 75, 3019-3030 (2003).

14. Blekherman, G. et al. Bioinformatics tools for cancer metabolomics. Metabolomics 7, 329-343 (2011).

15. Guijas, C. et al. METLIN: A Technology Platform for Identifying Knowns and Unknowns. Anal. Chem. $7 \mathrm{~b} 04424$ https://doi. org/10.1021/acs.analchem.7b04424 (2018).

16. Martina, B. E. E., Koraka, P. \& Osterhaus, A. D. M. E. Dengue virus pathogenesis: An integrated view. Clinical Microbiology Reviews 22, 564-581 (2009).

17. Gong, W. et al. Serum metabolomic profiling of piglets infected with virulent classical swine fever virus. Front. Microbiol. 8 (2017).

18. Murakami, M. Bioactive Lipid Mediators: Current Reviews and Protocols, https://doi.org/10.1007/978-4-431-55669-5 (2015).

19. Cui, L. et al. Serum Metabolome and Lipidome Changes in Adult Patients with Primary Dengue Infection. PLoS Negl. Trop. Dis. 7 (2013). 
20. Heaton, N. S. \& Randall, G. Dengue virus-induced autophagy regulates lipid metabolism. Cell Host Microbe 8, 422-432 (2010).

21. Mizushima, N., Yoshimori, T. \& Levine, B. Methods in Mammalian Autophagy Research. Cell 140, 313-326 (2010).

22. Levine, B. \& Kroemer, G. Autophagy in the Pathogenesis of Disease. Cell 132, 27-42 (2008).

23. Qian, M., Fang, X. \& Wang, X. Autophagy and inflammation. Clin. Transl. Med. 6, 24 (2017)

24. Singh, R. et al. Autophagy regulates lipid metabolism. Nature 458, 1131-1135 (2009).

25. Zhang, J. et al. Positive-strand RNA viruses stimulate host phosphatidylcholine synthesis at viral replication sites. Proc. Natl. Acad. Sci. 113, E1064-E1073 (2016).

26. Lee, W.-M. \& Ahlquist, P. Membrane synthesis, specific lipid requirements, and localized lipid composition changes associated with a positive-strand RNA virus RNA replication protein. J. Virol. 77, 12819-12828 (2003).

27. Martin, S. \& Parton, R. G. Lipid droplets: A unified view of a dynamic organelle. Nature Reviews Molecular Cell Biology 7, 373-378 (2006).

28. Carneiro, L. A. M. \& Travassos, L. H. Autophagy and viral diseases transmitted by Aedes aegypti and Aedes albopictus. Microbes Infect. 18, 169-171 (2016).

29. Sagan, S. M. et al. The influence of cholesterol and lipid metabolism on host cell structure and hepatitis C virus replication. Biochem Cell Biol 84, 67-79 (2006)

30. Stoeck, I. K. et al. Hepatitis C virus replication depends on endosomal cholesterol homeostasis. J. Virol. JVI.01196-17, https://doi. org/10.1128/JVI.01196-17 (2017)

31. Albulescu, L., Wubbolts, R., van Kuppeveld, F. J. M. \& Strating, J. R. P. M. Cholesterol shuttling is important for RNA replication of coxsackievirus B3 and encephalomyocarditis virus. Cell. Microbiol. 17, 1144-1156 (2015).

32. Benveniste, J. et al. Semi-synthesis and proposed structure of platelet-activating factor (PAF): PAF-acether an alkyl ether analog of lysophosphatidylcholine. C. R. Seances Acad. Sci. D. 289, 1037-1040 (1979).

33. Hanahan, D. J. Platelet activating factor: a biologically active phosphoglyceride. Annu. Rev. Biochem. 55, 483-509 (1986).

34. Gale, T. V., Horton, T. M., Grant, D. S. \& Garry, R. F. Metabolomics analyses identify platelet activating factors and heme breakdown products as Lassa fever biomarkers. PLoS Negl. Trop. Dis. 11 (2017).

35. Itabe, H. Oxidized phospholipids as a new landmark in atherosclerosis. Prog. Lipid Res. 37, 181-207 (1998)

36. Snyder, F. Platelet-activating factor: the biosynthetic and catabolic enzymes. Biochem. J. 305, 689 (1995).

37. Blank, M. L., Lee, Y. J., Cress, E. A. \& Snyder, F. Stimulation of the de novo pathway for the biosynthesis of platelet-activating factor (PAF) via cytidylyltransferase activation in cells with minimal endogenous PAF production. J. Biol. Chem. 263, 5656-5661 (1988).

38. Uozumi, N. et al. Role of cytosolic phospholipase A2 in allergic response and parturition. Nature 390, 618-622 (1997).

39. Shindou, H., Ishii, S., Uozumi, N. \& Shimizu, T. Roles of cytosolic phospholipase A(2) and platelet-activating factor receptor in the Ca-induced biosynthesis of PAF. Biochem. Biophys. Res. Commun. 271, 812-7 (2000).

40. Prescott, S. M., Zimmerman, G. A., Stafforini, D. M. \& McIntyre, T. M. Platelet-activating factor and related lipid mediators. Annu. Rev. Biochem. 69, 419-445 (2000).

41. Ishii, S. \& Shimizu, T. Platelet-activating factor (PAF) receptor and genetically engineered PAF receptor mutant mice. Prog Lipid Res 39, 41-82 (2000).

42. Liscovitch, M. \& Cantley, L. C. Lipid second messengers. Cell 77, 329-334 (1994).

43. Liu, Y. et al. Current Understanding of Platelet-Activating Factor Signaling in Central Nervous System Diseases. Molecular Neurobiology 54, 5563-5572 (2017).

44. Dent, G. et al. Protein kinase C inhibition enhances platelet-activating factor-induced eicosanoid production in human eosinophils. Am. J. Respir. Cell Mol. Biol. 18 (1998).

45. Liu, L. et al. Triple role of platelet-activating factor in eosinophil migration across monolayers of lung epithelial cells: eosinophil chemoattractant and priming agent and epithelial cell activator. J. Immunol. 161, 3064-70 (1998).

46. Roth, M. et al. Platelet-activating factor exerts mitogenic activity and stimulates expression of interleukin 6 and interleukin 8 in human lung fibroblasts via binding to its functional receptor. J Exp Med 184, 191-201 (1996).

47. Lou, Y. P. et al. Platelet-activating factor induces goblet cell hyperplasia and mucin gene expression in airways. Am. J. Respir. Crit. Care Med. 157, 1927-1934 (1998).

48. Souza, D. G. et al. Essential role of platelet-activating factor receptor in the pathogenesis of Dengue virus infection. Proc. Natl. Acad. Sci. USA 106, 14138-43 (2009).

49. Yeaman, M. R. Platelets: At the nexus of antimicrobial defence. Nature Reviews Microbiology 12, 426-437 (2014).

50. Shiraki, R. et al. Expression of Toll-like receptors on human platelets. Thromb. Res. 113, 379-385 (2004).

51. Solomon Tsegaye, T. et al. Platelet activation suppresses HIV-1 infection of T cells. Retrovirology 10 (2013).

52. Berthet, J. et al. Human platelets can discriminate between various bacterial LPS isoforms via TLR4 signaling and differential cytokine secretion. Clin. Immunol. 145, 189-200 (2012).

53. Anderson, H. A. \& Roche, P. A. MHC class II association with lipid rafts on the antigen presenting cell surface. Biochim. Biophys. Acta - Mol. Cell Res. 1853, 775-780 (2015).

54. Kallikourdis, M. et al. Phosphatidylinositol 4-Phosphate 5-Kinase $\beta$ Controls Recruitment of Lipid Rafts into the Immunological Synapse. J. Immunol. 196, 1955-1963 (2016).

55. Assinger, A. Platelets and infection - An emerging role of platelets in viral infection. Frontiers in Immunology 5 (2014).

56. Zapata, J. C., Cox, D. \& Salvato, M. S. The Role of Platelets in the Pathogenesis of Viral Hemorrhagic Fevers. PLoS Negl. Trop. Dis. 8 (2014).

57. Yang, K. D., Wang, C. L. \& Shaio, M. F. Production of cytokines and platelet activating factor in secondary dengue virus infections. J. Infect. Dis. 172, 604-605 (1995).

58. Costa, V. V., Fagundes, C. T., Souza, D. G. \& Teixeira, M. M. Inflammatory and innate immune responses in dengue infection: Protection versus disease induction. American Journal of Pathology 182, 1950-1961 (2013).

59. De Azeredo, E. L., Monteiro, R. Q. \& De-Oliveira Pinto, L. M. Thrombocytopenia in dengue: Interrelationship between virus and the imbalance between coagulation and fibrinolysis and inflammatory mediators. Mediators of Inflammation 2015 (2015).

60. Von Hundelshausen, P. et al. RANTES deposition by platelets triggers monocyte arrest on inflamed and atherosclerotic endothelium. Circulation 103, 1772-1777 (2001).

61. Rodenhuis-Zybert, I. A. et al. Immature dengue virus: A veiled pathogen? PLoS Pathog. 6 (2010).

62. Noisakran, S. et al. Role of CD61+ cells in thrombocytopenia of dengue patients. Int. J. Hematol. 96, 600-610 (2012).

63. Lin, C. F. et al. Generation of IgM anti-platelet autoantibody in dengue patients. J. Med. Virol. 63, 143-149 (2001).

64. Geoghegan, V. et al. Perturbed cholesterol and vesicular trafficking associated with dengue blocking in Wolbachia-infected Aedes aegypti cells. Nat. Commun. 8 (2017).

65. Colombo, T. E. et al. Dengue virus surveillance: Detection of DENV-4 in the city of São José do Rio Preto, SP, Brazil. Acta Trop. 164, $84-89$ (2016)

66. Xia, J. \& Wishart, D. S. Using metaboanalyst 3.0 for comprehensive metabolomics data analysis. Curr. Protoc. Bioinforma. 2016, 14.10.1-14.10.91 (2016).

67. Xia, J., Sinelnikov, I. V., Han, B. \& Wishart, D. S. MetaboAnalyst 3.0-making metabolomics more meaningful. Nucleic Acids Res. 43, W251-W257 (2015). 


\section{Acknowledgements}

CFORM would like to thank the São Paulo Research Foundation by the grant (16/17066-2). We also thank the Coordination for the Improvement of Higher Level Personnel (CAPES) for the fellowships from TMG (PROEX: 1489740) and MZD. DNO would like to thank the Brazilian Ministry of Health (Plano Nacional de Enfrentamento ao Aedes aegypti e à Microcefalia [Brazilian Plan for Fighting Aedes aegypti and Microcephaly]) for the fellowship under process No. 88887.137889/2017-00. JLPM and RRC thank MCTIC-CNPq/MEC-CAPES/ MS-Decit/FNDCT No. 14/2016 for the grant. MLN would like to thank the São Paulo Research Foundation for the grant (FAPESP, Process No. 13/21719-3). Finally, RRC would like to thank the São Paulo Research Foundation for the grant (FAPESP, Process Nos. 11/50400-0 and 15/06809-1).

\section{Author Contributions}

C.F.O.R.M. performed experiments, data collection, analyzed data and wrote the manuscript. M.Z.D. performed experiments and data collection. J.D., D.N.O. and T.M.G. analyzed data and revised the manuscript. T.E.C., M.L.N. and J.L.P.M. performed patient selection, diagnosis, sample collection and preparation and revised the manuscript. M.L.N., J.L.P.M. and R.R.C. coordinated the team, revised the manuscript and idealized all experiments.

\section{Additional Information}

Supplementary information accompanies this paper at https://doi.org/10.1038/s41598-018-30385-x.

Competing Interests: The authors declare no competing interests.

Publisher's note: Springer Nature remains neutral with regard to jurisdictional claims in published maps and institutional affiliations.

(c) Open Access This article is licensed under a Creative Commons Attribution 4.0 International

License, which permits use, sharing, adaptation, distribution and reproduction in any medium or format, as long as you give appropriate credit to the original author(s) and the source, provide a link to the Creative Commons license, and indicate if changes were made. The images or other third party material in this article are included in the article's Creative Commons license, unless indicated otherwise in a credit line to the material. If material is not included in the article's Creative Commons license and your intended use is not permitted by statutory regulation or exceeds the permitted use, you will need to obtain permission directly from the copyright holder. To view a copy of this license, visit http://creativecommons.org/licenses/by/4.0/.

(C) The Author(s) 2018 